\title{
Editorial
}

\section{Modeling for Prognostics and Health Management: Methods and Applications}

\author{
Xiao-Sheng Si, ${ }^{1}$ Chang-Hua Hu, ${ }^{1}$ Enrico Zio, ${ }^{2,3}$ and Gang $\mathrm{Li}^{4}$ \\ ${ }^{1}$ Xi'an Institute of High-Tech., Xian, Shaanxi 710025, China \\ ${ }^{2}$ Energy Department, Politecnico di Milano, Via Ponzio 34/3, 20133 Milan, Italy \\ ${ }^{3}$ Chair on System Science and Energetic Challenge, European Foundation for New Energy, Electricite de France, \\ Ecole Centrale and Supelec, Grande Voie des Vignes, Chatenay-Malabry, 92295 Paris Cedex, France \\ ${ }^{4}$ University of Southern California, Los Angeles, CA 90089, USA
}

Correspondence should be addressed to Xiao-Sheng Si; sixiaosheng@gmail.com

Received 22 April 2015; Accepted 22 April 2015

Copyright (C) 2015 Xiao-Sheng Si et al. This is an open access article distributed under the Creative Commons Attribution License, which permits unrestricted use, distribution, and reproduction in any medium, provided the original work is properly cited.

Introduction. Prognostics and health management (PHM) can make full use of condition monitoring $(\mathrm{CM})$ data from a functioning system to assess the reliability of the system in its actual life cycle conditions, to determine the advent of failure and to mitigate system risk through managerial activities. PHM is a systematic approach that is used to evaluate the reliability of a system in its actual life-cycle conditions, predict failure progression, and mitigate operating risks via management actions. There are two parts in PHM, namely, "prognostics" and "health management". Prognostics is often characterized by estimating the remaining useful life (RUL) of a system using available CM information. Once such prognosis is available, appropriate health management actions such as repair, replacement, and logistic support can be performed to achieve the required system's operational objectives. A requirement of a PHM enabled system is the ability to estimate the RUL, which can provide the decision-maker with enough lead-time to perform the necessary maintenance actions prior to failure. This prognostic ability is a fundamental prerequisite for health management. So far, estimating the RUL, conditional on the CM data, has been considered as one of the most central components in PHM and attached great importance in practice.

With advances in information and sensing technologies, degradation signals of the system can be obtained relatively easily through CM techniques. However, it is quite common in practice that the degradation occurs in a stochastic way for a number of engineering systems such as bearings, gyroscopes, and battery systems. As a result, the RUL is also a random variable, resulting in the difficulty to estimate the RUL with certainty. The past decade has witnessed an increasingly growing research interest on various aspects of stochastic degradation-modelling from the observed signals for prognostics. This is partly caused by its importance in a variety of fields such as maintenance, inventory control, public health surveillance and management, and more.

The main focus of this special issue will be on the new theories and methodologies and their applications in fault diagnosis, degradation modeling, and prognostics and health management for complex engineering systems, especially in industry applications. The special issue enables researchers worldwide to report their most recent developments and ideas in the field, with a special emphasis on technical advances and new trends within the last five years.

In the next section, we give a brief description of the papers in this special issue.

An Overview of the Special Issue. This special issue comprises twenty-one papers, which are carefully selected from many submissions by a rigorous peer review process. The contents of which are summarized as follows.

"Remaining Useful Lifetime Prognosis of Controlled Systems: A Case of Stochastically Deteriorating Actuator" by D. $\mathrm{N}$. Nguyen et al. proposed a novel method to address the case of automatic controlled systems which deteriorate during its operation because of components' wear or deterioration. 
This paper considers a controlled system with ProportionalIntegral-Derivative controller. It is assumed that the actuator is subject to shocks that occur randomly in time. An integrated model is proposed to jointly describe the state of the controlled process and the actuator deterioration. By considering a Piecewise Deterministic Markov Process, the remaining useful lifetime of the system can be estimated by a two-step approach. In the first step referred as the "Diagnostic" step, the system state is estimated online from the available monitoring observations by using a Particle Filtering method. In the second step referred as the "Prognostic" step, the remaining useful lifetime is estimated as a conditional reliability by Monte Carlo Simulation.

"Recursive Gaussian Process Regression Model for Adaptive Quality Monitoring in Batch Processes" by L. Zhou et al. proposes an adaptive monitoring scheme based on the recursive Gaussian process (RGP) model. Based on the initial data, a Gaussian process model and the corresponding SPE statistic are constructed at first. When the new batches of data are included, a strategy based on the RGP model is used to choose the proper data for model updating. The performance of the proposed method is finally demonstrated by a penicillin fermentation batch process and the result indicates that the proposed monitoring scheme is effective for adaptive modeling and online monitoring.

"A Well-Designed Parameter Estimation Method for Lifetime Prediction of Deteriorating Systems with Both Smooth Degradation and Abrupt Damage" by C. Yu and C. Jiang models the degradation trajectory of the deteriorating system by a random coefficient regression (RCR) model with positive jumps, where the RCR part is used to model the continuous smooth degradation of the system and the jump part is used to characterize the abrupt damages due to random shocks. Based on a specified threshold level, the probability density function and cumulative distribution function of the lifetime can be derived analytically. The unknown parameters associated with the derived lifetime distributions can be estimated via a well-designed parameter estimation procedure on the basis of the available degradation recordings of the deteriorating systems.

"Circuit Tolerance Design using Belief Rule Base" by X.-B. $\mathrm{Xu}$ et al. constructs a belief rule basis (BRB) system to model the highly nonlinear relationship between circuit component parameters and the performance of the circuit by utilizing available knowledge from circuit simulations and circuit designers. By using rule inference in the BRB system and clustering analysis, the acceptability regions of the component parameters can be separated from the value domains of the component parameters. Using the established nonlinear relationship represented by the BRB system, an optimization method is proposed to seek the optimal feasibility region in the acceptability regions so that the volume of the tolerance region of the component parameters can be maximized.

"Reliability Analysis of Load-Sharing K-Out-of-N System considering Component Degradation" by J. Guo et al. proposes a method combining a tampered failure rate model with a performance degradation model to analyze the reliability of load-sharing $K$-out-of- $N$ system with degrading components. The proposed method considers the value of $K$ as a variable which is derived by the performance degradation model. The load-sharing effect is evaluated by the tampered failure rate model. Monte-Carlo simulation procedure is used to estimate the discrete probability distribution of $K$.

"A Novel Strong Tracking Fault Prognosis Algorithm" by Q. Zhang et al. presents a novel strong tracking fault prognosis algorithm to settle the problem of abruptly changing states and the degeneracy in particle filter algorithm. In the proposed algorithm, the artificial immunity algorithm is first introduced to resolve the degeneracy problem, and then the strong tracking filter is introduced to enhance the ability to track abruptly changing states. The particles are updated by strong tracking filter, and better particles are selected by utilizing the artificial immune algorithm to estimate states.

"Generalized Accelerated Failure Time Frailty Model for Systems Subject to Imperfect Preventive Maintenance" H. Yin et al. introduces the models in the field of survival analysis into condition-based maintenance. Specifically, the generalized accelerated failure time frailty model is investigated to model the failure likelihood of industrial systems. Further, on the basis of the maximum likelihood (ML) estimation and expectation maximization (EM) algorithm, the hybrid MLEM algorithm is investigated for the estimation of parameters. The performance of the model is analyzed through the prediction of remaining useful life using the testing-data.

"The Assessment and Foundation of Bell-Shaped Testability Growth Effort Functions Dependent System Testability Growth Models Based on NHPP” by T.-M. Li et al. investigates a type of STGM (system testability growth model) based on the nonhomogeneous Poisson process which incorporates TGEF (testability growth effort function). First, the process of TGT (testability growth test) for equipment is analyzed to show that the TGT can be divided into two steps: make the unit under test be in broken condition to identify TDL (testability design limitation) and remove the TDL. The amount of TGF (testability growth effort) spent on identifying TDL is a crucial issue which decides the shape of testability growth curve and that the TGF increases firstly and then decreases at different rate in the whole life cycle. Further, five TGEF are included, including an Exponential curve, a Rayleigh curve, a logistic curve, a delayed S-shape curve, or an inflected Sshaped curve which are collectively referred to as Bell-shaped TGEF into STGM.

"Fault Prediction Algorithm for Multiple Mode Process Based on Reconstruction Technique" by J. Ma and J. Xu studies the problems of multiple mode process fault detection, fault estimation, and fault prediction systematically based on multi-PCA model in the framework of fault reconstruction technique. A multi-PCA model is used for fault detection in steady state process under different conditions while a weighted algorithm is applied for transition process. Then, the proposed method describes the faults quantitatively and uses the optimization method to derive the fault amplitude under the sense of fault reconstruction. Finally, support vector machine is used to predict the trend of the fault amplitude.

"A BRB Based Fault Prediction Method of Complex Electromechanical Systems" by B. Zhang et al. constructs a new fault prognosis model based on the basis of belief 
rule base to utilize both the quantitative information and qualitative knowledge. Further, an evidential reasoning based optimal algorithm is developed to train the established fault prediction model. As such, the fault prediction accuracy can be improved effectively.

"Anomaly Detection and Degradation Prediction of MOSFET" by L. F. Wu et al. analyzes the failure models and mechanisms of MOSFETs. Based on the experimental data, a nonlinear dual-exponential degradation model for MOSFETs is presented. Then, an approach for MOSFET degradation state prediction is provided by using a strong tract filter based on the obtained degradation model.

"A Belief Rule-Based Safety Evaluation Approach for Complex Systems" by J. Zhang et al. proposes a generic belief rule-based safety evaluation approach for large-scale complex systems. In the proposed method, the system is firstly decomposed and filtered into the measurable attributes that may potentially contribute to the uncertainty. Then, a belief rule base is established with all antecedents, consequents, and attributes presented in belief degrees to estimate the uncertainty with a distribution representation.

"Fault Diagnosis in Condition of Sample Type Incompleteness using Support Vector Data Description" by H. Yi et al. discusses the relationship between sample-type incompleteness and the classifier-based diagnostic accuracy of complicated systems. Then, a support vector data descriptionbased approach is proposed to refine the construction of fault regions and increase the diagnostic accuracy for the condition of incomplete sample types. In the proposed method, the effect of sample-type incompleteness is taken into consideration.

"A Fault Diagnosis Method of Power Systems Based on Gray System Theory" by H. Darong et al. presents a new model for identifying fault component by using gray theory to provide some decision-making suggestions for fault diagnosis of power systems. In the proposed model, the recognition algorithm of the power supply interrupted districts and the assignment principle of fault state vectors are depicted according to the working principle of protective relays and circuit breakers. Based on the concept of the Gray correlation degree, the fault information explanation degree model is constructed and the judging method of malfunction and rejection for PRs and CBs is established. To achieve the goal of the fault diagnosis, the fault diagnosis procedure is designed for the concerned power systems.

"Incipient Gearbox Fault Diagnosis Based on the Reverse State Transformation of the Chaotic Duffing Oscillator and Sampling Integral Technology" by J. Li and J. M. Zhao presents a method based on chaos theory and sampling integral technology to detect the incipient fault of gearbox according to the characters of the gearbox vibration signals. Sampling integral technology was used to improve the tracking ability of fault signals with lower signal-to-noise ratio. The small changes in the sidebands of meshing frequency can be detected by the transformation of chaotic phase diagram, and then the incipient faults can be diagnosed.

"A Fault Diagnosis Approach for Gas Turbine Exhaust Gas Temperature Based on Fuzzy C-Means Clustering and Support Vector Machine" by Z.-T. Wang et al. proposes a fusion approach based on Fuzzy C-Means (FCM) clustering algorithm and Support Vector Machine (SVM) classification model. FCM clustering algorithm is used to realize clustering analysis and obtain the state pattern. SVM multiclassification model is designed to carry out the state pattern recognition and fault diagnosis.

"A Diagnosis Method for Rotation Machinery Faults Based on Dimensionless Indexes Combined with K-Nearest Neighbor Algorithm" by J. Xiong et al. presents a diagnosis method for rotation machinery fault based on dimensionless indexes combined with $K$-nearest neighbor ( $\mathrm{KNN}$ ) algorithm. This method uses a $K N N$ algorithm and an evidence fusion theoretical formula to deal with fuzzy data, incomplete data, and accurate data. The input information is integrated by an evidence synthesis formula to get the diagnosis result.

"Fault Sample Generation for Virtual Testability Demonstration Test Subject to Minimal Maintenance and Scheduled Replacement" by Y. Zhang et al. describes the fault occurrence process by stochastic process theory and discusses the fault occurrence process subjected to minimal repair modeled by nonhomogeneous Poisson process (NHPP). The interarrival time distribution function of the next fault event is proposed and three typical kinds of parameterized NHPP are discussed. Finally, the procedure of fault sample generation is put forward with the assumptions of minimal maintenance and scheduled replacement. As such, the fault modes and their occurrence time subject to specified conditions and time period can be obtained.

"Research on FCM and NHL Based High Order Mining Driven by Big Data" by Z. Peng et al. proposes an FCM and NHL based high order mining algorithm to get the high order evaluation and correlation degree among big data with the characteristics of multidimension and multigranularity. The algorithm is applied in scientific and technical talent forecast.

"The Robust Passive Location Algorithm for Maneuvering Target Tracking" by X. Yang et al. proposes a robust passive location algorithm based on the orthogonality principle and the fuzzy extended Kalman filter to track the maneuvering target in the uncertain passive location system. Different from other passive location method, the noise is described by the trapezoidal possibility distributions rather than the Gaussian probability distributions.

"Attitude Estimation Based on the Spherical Simplex Transformation Modified Unscented Kalman Filter" by J. Zhao et al. proposes an antenna attitude estimation algorithm to improve the antenna pointing accuracy for the satellite and the estimation is achieved by the spherical simplex transformation modified UKF. The extrapolated angle accelerator improves the real-time character of the system. Besides, the antijamming capacity of the system has been increased for the judgment rules of the motion state.

Concluding Remarks. Due to the limited space, we cannot introduce all the papers in this special issue with more details. Of course, the selected topics and papers are not a comprehensive representation of the area of this special issue. However, we do hope that this special issue contains useful information that can help motivate more researchers to contribute to this fascinating area. 


\section{Acknowledgments}

We would like to express appreciation to the authors for their excellent contributions and patience in assisting us. We also appreciate all reviewers who dedicated their time and effort to perform review of all the submitted papers and provided valuable suggestions to the authors.

Xiao-Sheng Si

Chang-Hua $\mathrm{Hu}$

Enrico Zio

Gang Li 


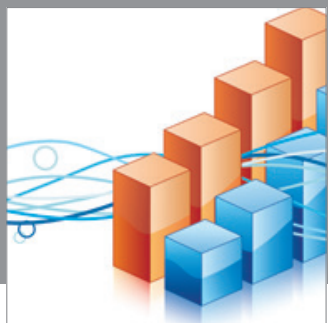

Advances in

Operations Research

mansans

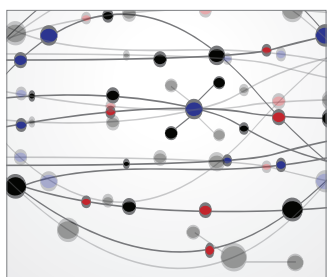

The Scientific World Journal
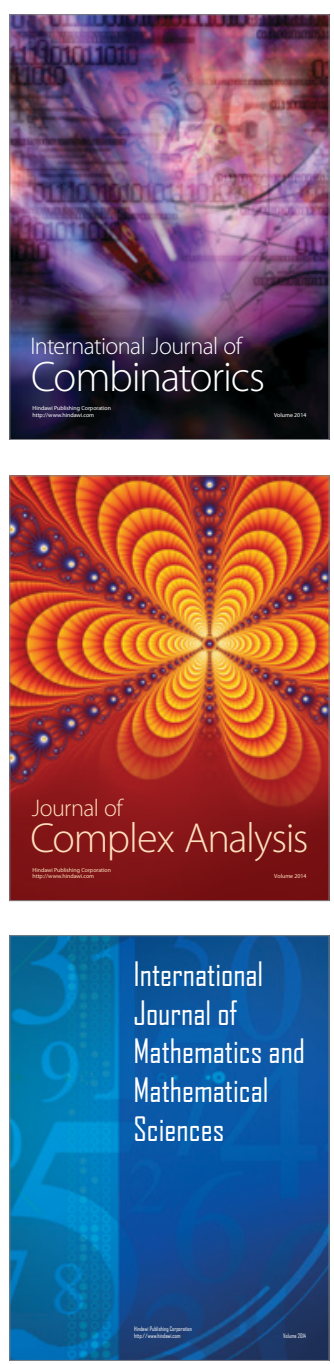
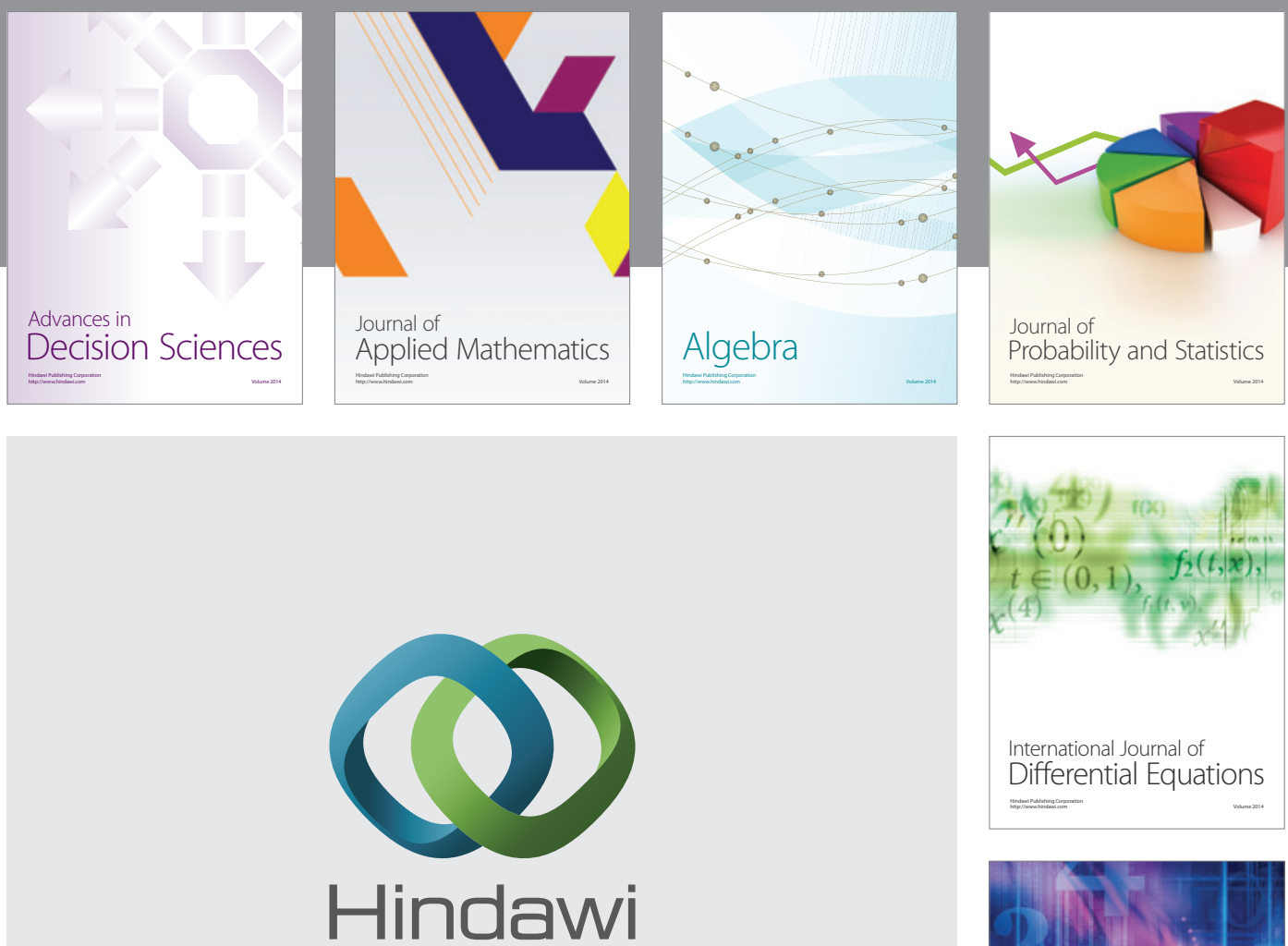

Submit your manuscripts at http://www.hindawi.com
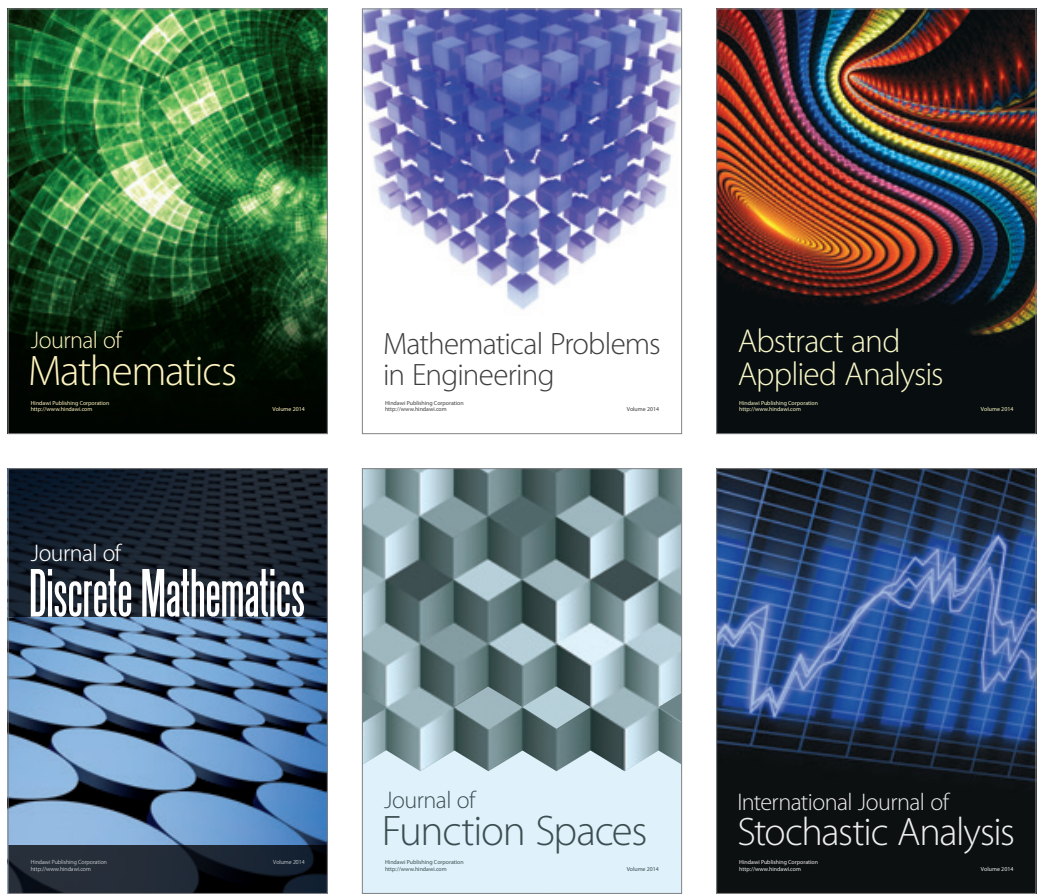

Journal of

Function Spaces

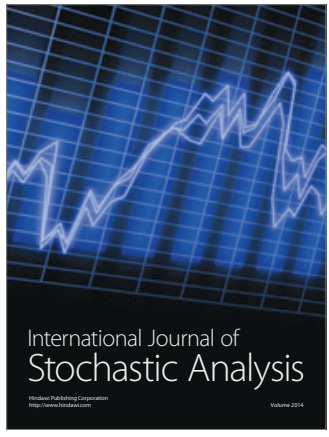

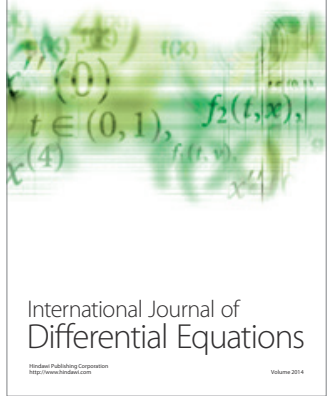
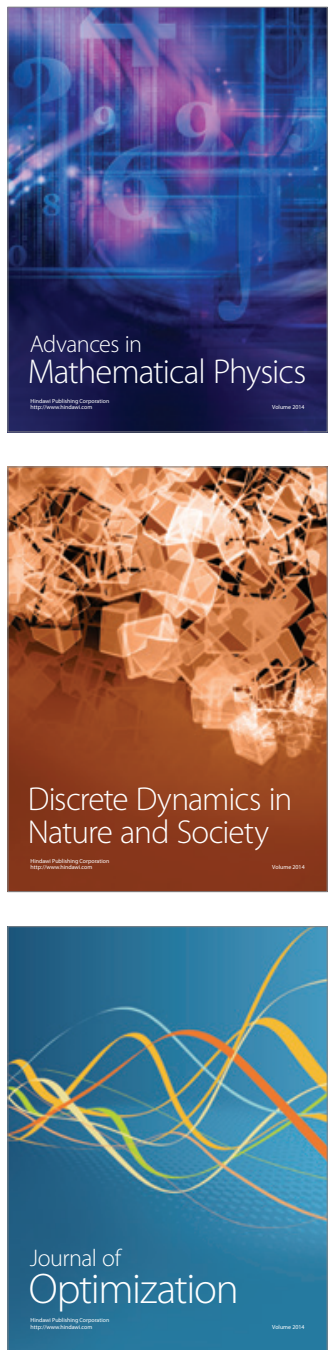